DEPARTMENT OF THE NAVY

Bureau of Ships

Contract Nonr-220(12)

\title{
Water Tunnel Techniques for Force Measurements on Cavitating Hydrofoils
}

\author{
BLAINE R. PARKIN \\ ROBERT W. KERMEEN
}

Hydrodynamics Laboratory

California Institute of Technology

Pasadena, California 


\title{
Water Tunnel Techniques for Force Measurements on Cavitating Hydrofoils'
}

\author{
By Dr. Blaine R. Parkin ${ }^{2}$ and Robert W. Kermeen²
}

The paper reviews briefly experimental techniques and data-correction procedures developed in connection with studies in the High Speed Water Tunnel, California Institute of Technology, for hydrofoil force measurements. The matters discussed relate chiefly to problems of force measurement in cavitating flow and to procedures devised to solve them. Items considered in this connection are water-tunnel balances, the effects of tip-clearance flows, tare corrections, the importance of cavity-pressure measurements, cavitation scaling, tunnel-wall effects, and hydroelastic effects.

The use of the water tunnel as an aid in the design and development of marine propellers has long been an established practice in naval architecture. It now appears that there are other important areas in which such a facility can supplement the towing tank effectively. One such area of investigation for which the water tunnel is particularly well suited is the study of the hydrodynamics of hydrofoil sections. For this reason the Bureau of Ships through its Fundamental Research Program, David Taylor Model Basin, has sponsored a hydrofoil research program at the California Institute of Technology. The purpose of the present paper is to review briefly some of the more important procedures which have been used for hydrofoil force measurements in the High Speed Water Tunnel.

It is generally recognized that water-tunnel testing and low-speed wind-tunnel testing have many features in common. Indeed, many procedures which have been found useful in wind-tunnel experiments may be used without alteration in water-tunnel test work. These experimental procedures and data-correction methods are well known and there is a considerable body of literature, mainly from the field of aeronautical testing, con-

${ }_{1}$ This paper summarizes one aspect of a hydrofoil research project carried out under the Bureau of Ships fundamental hydrodynamics research program, Project NS 715-102, David Taylor Model Basin. Reproduction in whole or in part is permitted for any purpose of the United States Government.

'California Institute of Technology, Hydrodynamies Laboratory, Pasadena, Cal.

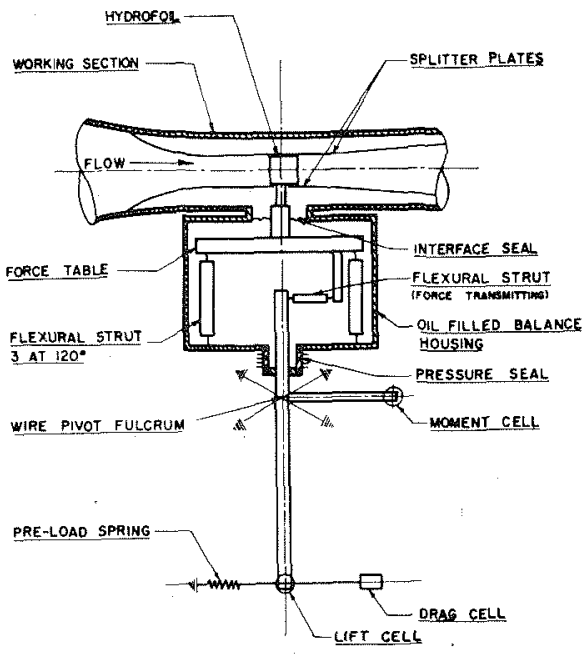

Fig. 1 Schematic of three-component force balance installed for hydrofoil force measurements

cerning them. To review these well-established results here would be superfluous. Instead, the purpose of this paper is to consider those methods which have been developed to solve the particular experimental requirements of hydrofoil testing in the High Speed Water Tunnel. Probably the most important of these requirements-the only requirement which has necessitated experimental methods differing greatly from standard wind-tunnel techniques-is that the forces on the profile be measured in cavitating flow. The consequences of this fact form the theme of this paper.

\section{The Force-Measuring System}

In wind-tunnel practice one of ten determines the lift on an airfoil section by integrating the static pressure distribution along the floor and ceiling of the test section with the help of an integrating manometer. Similarly, the drag is customarily obtained from measurements of the loss in total pressure of the air in the wake of the profile (1). ${ }^{3}$ These techniques would be equally applicable to profile force measurements in water tunnels were it not for the necessity of measuring these forces in cavitating as well as in noncavitating flows. The use of static pressure taps in regions of very low pressure in a water flow

\footnotetext{
${ }^{3}$ Numbers in parentheses refer to the Bibliography at the end of the paper.
} 
is not always permissible because water in the pressure taps usually cavitates so that the resulting manometer indications are meaningless. Therefore the forces and moments on a cavitating profile must be measured directly by an appropriate balance.

The design of a force balance to meet all conditions of water-tunnel operation is complicated by the severe requirements which a pressure seal must satisfy. In contrast to many low-speed wind tunnels, water tunnels used in cavitation work are at times operated at static pressures in the test section as great as 100 psi above atmospheric pressure. These high pressures introduce very large axial forces of the order of 500 to $1000 \mathrm{lb}$ from the seal into the balance structure. Yet the seal must be designed or located so as not to introduce a force greater than $1 / 100,000$ of this axial load into the hydrodynamic force-measuring system. Similar, although not so severe, requirements must be met when cavitation is being produced in the test section. In this case the static pressure in the tunnel is usually significantly less than the outside atmospheric pressure and now the seal force, to be isolated from the measuring system, has a direction opposite to the axial force just considered.

A number of balances providing solutions to the pressure-seal problem have been designed and built both in this country and abroad. A comparison of these balances and of the various ways in which the seal problem has been attacked is clearly beyond the scope of the present paper. For the work carried out in the High Speed Water Tunnel a successful balance which overcomes the difficulties introduced by the pressure seal is shown schematically in Fig. 1 (2). The hydrodynamic reactions from the hydrofoil are resolved by the parallelogram mounted force table into lift, drag, and pitching moment. These components are transmitted from the force table to three hydraulic sensing cells by means of a lever. This lever is restrained from rotating or twisting about its fulcrum, by the lift, drag, and pitching-moment cells. The restraining forces in these cells are supplied by hydraulic pressures which are transmitted to three automatic beam balances. The balances indicate the magnitudes of the three components. The pressure seal which is a molded neoprene tube with metal-ring reinforcements is attached to the spindle close to the universal pivot center so that axial forces which might affect the lift and drag readings are grounded effectively. The seal is designed to have low torsional stiffness so that it has small influence on the moment readings. The design loading ranges for this balance are as follows:

$$
\begin{array}{rlr}
\text { Lift and Drag } & +150 \mathrm{lb} \\
-150 \mathrm{lb} & \text { Moment } & +560 \mathrm{in}-\mathrm{lb} \\
-560 \mathrm{in}-\mathrm{lb}
\end{array}
$$

A detailed analysis of the balance design and its performance is given in reference (2).

The use of a balance for direct measurements of profile characteristics requires that the model cannot touch the sides of the test section. On the other hand, from the theory of inviscid incompressible flows it is known that the lift distribution on an untwisted prismatic airfoil of

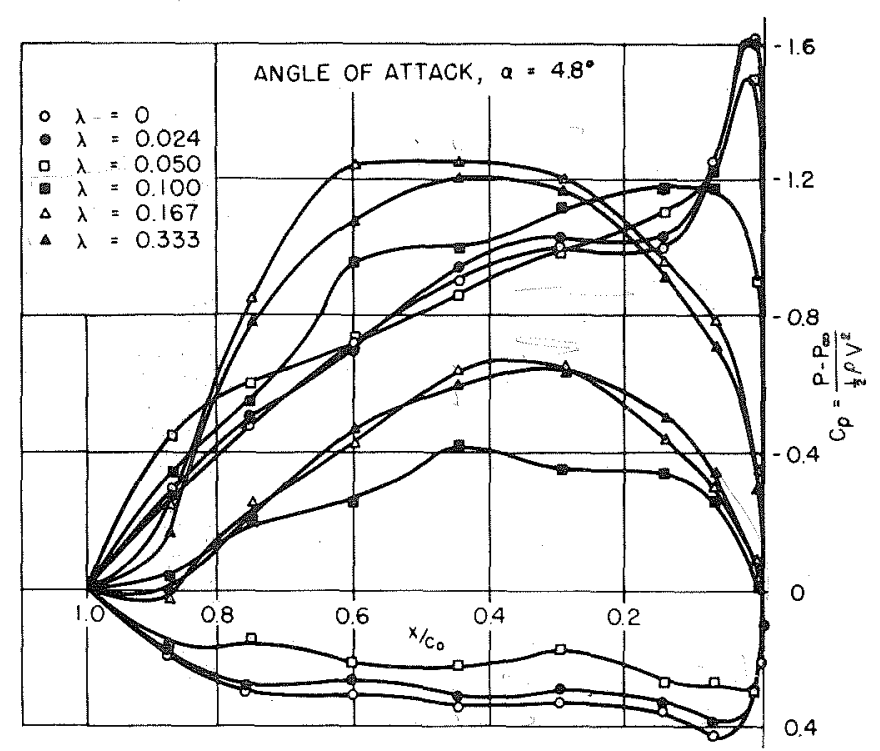

Fig. 2 Wall pressure distribution near the tip of an NACA 4412 hydrofoil (after Rains)

rectangular planform is independent of spanwise position if the airfoil spans a closed throat test section of almost any cross-sectional shape (3). This result has been found to hold in real fluids of small viscosity even though secondary flows originate in the test-section boundary layer at the junctures of the airfoil and the tunnel walls. For two-dimensional tests in rectangular channels it has long been known that, aside from possible boundary-layer contamination, these secondary flows have practically no effect on the pressure distribution over the central portions of the model and that the two-dimensional pressure distribution is transmitted essentially unchanged through the wall boundary layer. However, if there is a gap between the end of the hydrofoil and the test-section wall, potential-flow theory warns one that even small clearances could have pronounced effects upon the hydrodynamic characteristics of the hydrofoil, and one would suspect that the balance readings might not correspond to two-dimensional characteristics.

Even though real fluids allow appreciable end clearance flows through the gap, it turns out that their effects are not as severe as would be predicted by potential theory. For example, Rains (4) has measured the wall pressure distribution near the tip of a hydrofoil for a Reynolds number of 50,000 based on free-stream velocity and profile-chord length. His measurements were made for various values of the ratio of tip clearance to maximum profile thickness $\lambda$, and some of his results are reproduced in Fig. 2. This figure shows how the lift is maintained up to the wall for zero tip clearance and how the wall pressures change gradually from the $\lambda=0$ distribution with increasing values of $\lambda$ up to $\lambda=0.05$. For values of $\lambda$ greater than 0.05 the wall pressure distributions change rapidly with increasing tip clearance. Evidently for $\lambda$ $<0.05$ the flow in the clearance space is dominated by 


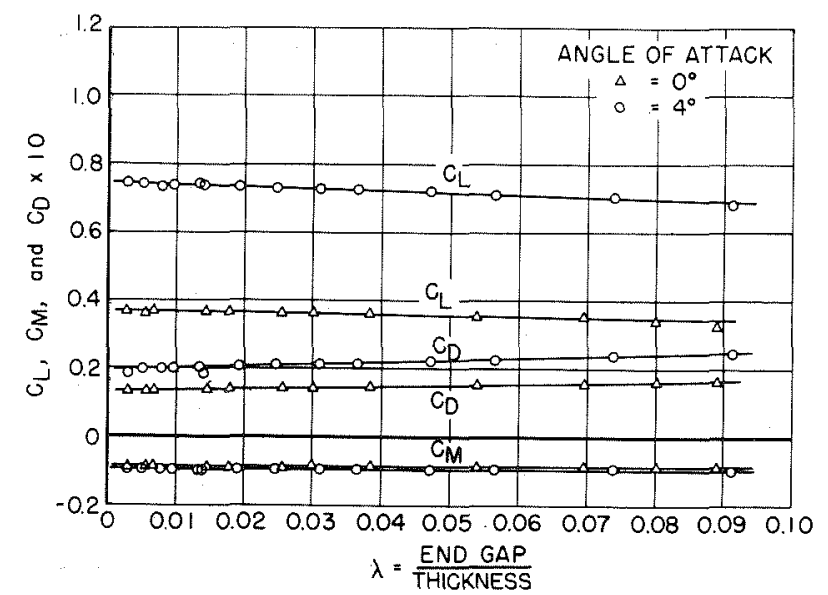

Fig. 3 Effect of end gap on the forces of an NACA 4412 hydrofoil in noncavitating flow at angles of attack of 0 and 4 deg

viscous forces and the pressure difference across the profile thickness is almost completely balanced by viscous resistance to the clearance flow. These results suggest, in the case of hydrofoil force measurements, that the tip gap should be maintained at a value which will insure essentially viscous clearance flows. Gaps satisfying this requirement necessarily must be small compared to the hydrofoil span. Therefore, one might expect the influence of end gaps on the total force on the hydrofoil to be small because it seems that their effects should be limited mainly to the immediate vicinity of the clearance flows. That such is the actual state of affairs is shown by the low slopes of the curves in Fig. 3 where lift, drag and pitching-moment measurements for the NACA 4412 hydrofoil are plotted for various values of the clearance ratio $\lambda$. The shallow slope of these curves would indicate that it is possible to keep the errors due to tip-clearance flows within 1 per cent of the indicated force or moment and still have end gaps which are large enough for practical tunnel operations. A close agreement between High Speed Water Tunnel measurements and twodimensional wind-tunnel measurements on the NACA 4412 at comparable Reynolds numbers substantiates these findings (5).

So far no attention has been given to the effects of tipclearance flows on the measurement of forces for fully cavitating hydrofoils. In this case the pressure difference across the profile thickness is reduced from its value corresponding to noncavitating flow so that one would expect the clearance flow to diminish as the cavitation increases. Accordingly, if the gap is set to a value which is acceptable for force measurements in noncavitating flow, it seems likely that this tip clearance will be found satisfactory for experiments on super-cavitating hydrofoils. This reasoning certainly appears plausible in the case of fully cavitating sharp-edged hydrofoils where very good agreement was obtained between Wu's twodimensional theory (6) and High Speed Water Tunnel experiments (7).
Finally, it must be admitted that the situation in regard to gap effects on incipient or partial cavitation is not as clear cut as in the two eases discussed previously. In this intermediate case cavitation forms prematurely at the intersections of the hydrofoil with the walls. As the cavitation develops on the foil proper the cavitation from the intersections spreads out to contaminate a larger area of the flow adjacent to the ends of the hydrofoil. It appears that this effect can be minimized mainly by using hydrofoils of long span because the effect exists at the intersections with or without gaps, although the gap size does modify it. Of course, the use of long-span models to minimize end effects introduces large forces which may be too large for existing balances to handle over suitable ranges of lift coefficient and free-stream velocity, or in many instances the hydrofoil sections may not be strong enough to withstand the additional loads imposed by a longer span. Certainly this matter needs clarification if we are to determine the best compromise between these conflicting requirements in this intermediate range of cavitation development.

\section{Tares}

The end gaps necessary to insure freedom from restraints of the balance-force table are not the only sources for error in the hydrodynamic-force indications. Related to this are the spurious reactions which arise from parts of the model and its mounting structure which are exposed to the flow but which are not a part of the hydrofoil proper. These extraneous forces may be large and must be subtracted from the total measured force readings. The large lift forces to which the hydrofoil models are subjected and the need to avoid spindle cavitation dictate the spindle-mounting configuration. Mounting the hydrofoil model on a circular disk set flush in the working-section wall eliminates one wall-clearance gap, reduces cavitation at the spindle-attachment point, and gives the maximum strength. For thin hydrofoils, in fact, it has been found necessary to machine the model and mounting disk from a solid piece of high-strength steel. The mounting disk must be large enough so that it extends beyond the region of steep pressure gradients around the hydrofoil model in order to reduce flow and cavitation in the radial-clearance gap around the disk.

In the High Speed Water Tunnel a mounting-disk diameter of approximately 1.5 times the hydrofoil chord was found to be adequate to prevent spurious cavitation in the radial-clearance gap under most operating condjtions (5). With such a large disk the viscous forces on the support structure become a large part of the total measured drag force. The forces on the disk in noncavitating flow are very nearly equal to that calculated for a flat plate. Since the hydrofoil is tested with varying amounts of cavitation, the only means of correcting the data for these extraneous forces is to measure them directly. This requires a series of disk tare-calibration runs for each hydrofoil tested. The forces on the mounting disk are measured by mounting the hydrofoil model from the tunnel wall opposite the force balance with a small clearance 
between the end of the hydrofoil and a dummy mounting disk attached to the spindle (5). The same test conditions are repeated with this arrangement as for the hydrofoil and mounting disk combination. The lift and pitching-moment disk tare forces are generally negligibly small. On the other hand, the drag on the mounting disk may be as much as 50 per cent of the total measured drag on the hydrofoil-disk combination at small attack angles. The accurate determination of this tare force is essential. When cavitation develops on the foil it causes the hydrofoil drag to increase, and, since the cavitation from the hydrofoil also covers a portion of the mounting disk, the tare drag becomes proportionately a much smaller portion of the total drag.

\section{Cavify Pressure Measuremenłs}

Another precaution which has been found necessary to insure meaningful force-balance indications in full cavity flows is that of measuring the cavity pressure while the forces are being measured. This information is essential if the results obtained are to be correlated with similar data from other facilities and if the designer is to use the tunnel results correctly. The justification for this procedure which has been followed in the recent High Speed Water Tunnel investigations $(5,7,8)$ originates from the well-known work of Reichardt (9), which showed that the cavity geometry and the drag force for a given axially symmetric body in full cavity flow is determined by the cavitation number based on cavity pressure.

The one important fact as far as the present discussions are concerned is that even in cavity flows which have not been produced artificially by air injection, the cavity pressure is not generally equal to the vapor pressure of the water. An example of this fact is shown in Fig. 4. In this illustration the cavitation number $K_{k}$ based on the measured cavity pressure is plotted against the cavitation number $K_{v}$ based upon the vapor pressure." These data, taken from an NACA 4412 hydrofoil in full cavity flow, show how the discrepancy between $K_{y}$ and $K_{k}$ increases as the cavitation numbers increase; that is, as the cavities become shorter. For these higher values of cavitation number the re-entrant jet at the rear of the short cavity causes the cavity to become a foamy mixture of air and water, thereby making reliable pressure measurements difficult to obtain. However, careful experimental techniques do permit consistent pressure measurements in this flow regime. These measurements indicate that the cavity pressure for such flows is several times greater than the vapor pressure of water. For lower values of the cavitation number the flow contains longer clear cavities which are filled with water vapor and with air which has diffused through the cavity wall from the water. In this case the cavity pressure seems to be constant within the range of cavitation numbers for which such clear gas and vapor-filled cavities exist.

As indicated previously, use of the wrong basis in computing the cavitation number for the representation of hydrofoil-force data can lead to appreciable errors in its interpretation. An example of the kind of discrepancies

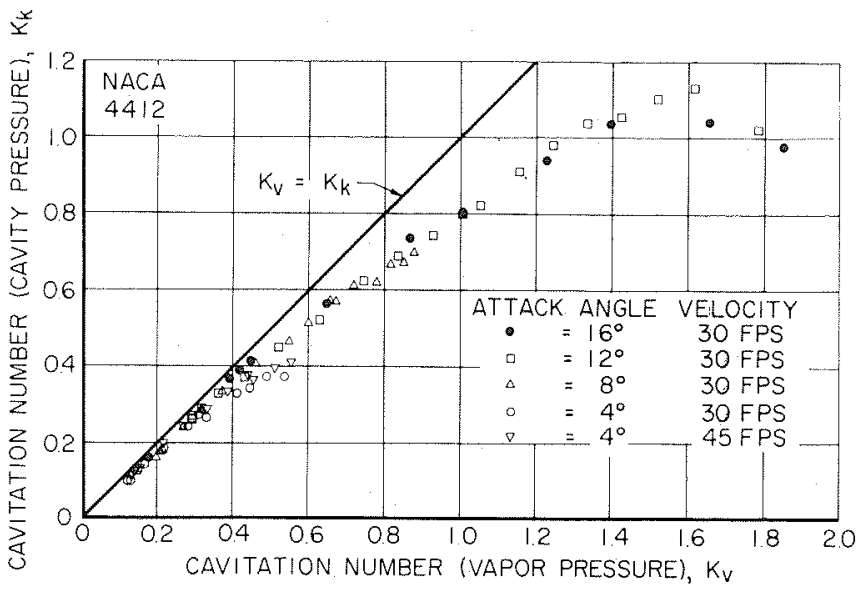

Fig. 4 Comparison between the cavitation number based on vapor pressure $K_{v}$ and cavitation number based on measured cavity pressure $K_{k}$ for well-developed cavitation

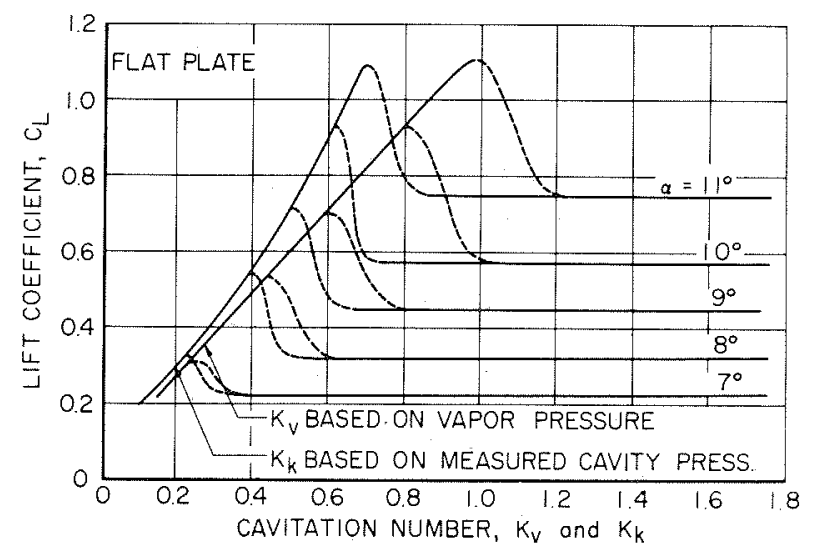

Fig. 5 Effect of different cavitation numbers on appearance of lift data

which are possible is shown in Fig. 5. In this figure the lift coefficient for a flat-plate hydrofoil is shown by curves which have been faired through a considerable number of data points. For clarity, these points have been omitted in the illustration. These data which were taken at several attack angles over a large range of cavitation numbers were plotted first as a function of the cavitation number $K_{v}$ and then wherever possible they were replotted as a function of the cavitation number $K_{k}$. This procedure resulted in the two families of curves shown for cavitation numbers which correspond to cavitating flows. If for the reasons just given the curves based on $K_{k}$ are considered to give the more nearly correct representation of the data, then in applications of the data to design situations involving full cavity flows serious errors in the estimation of the lift coefficient could result if the parameter $K_{v}$ is used as a basis for design. Fortunately we see from both Figs. 4 and 5 that significant errors result from the use of $K_{v}$ only for the higher cavitation numbers. For cavitation numbers below 0.3 , 
say, it would appear that the use of either $K_{v}$ or $K_{k}$ might be expected to give results within engineering accuracy.

Finally, it is important to note that one might suspect that pressure measurements at higher cavitation numbers, for which the cavity is a foamy mixture of air and water, might not be meaningful even though they are consistent. The fact is that the cavity for such flows is actually a mixture of air and water in highly unsteady motion. Accordingly any "consistent" pressure measurement represents some sort of a time average of these fluctuating pressures. Similarly one also expects that the measured hydrofoil forces represent an average of nonsteady forces which match the base-pressure fluctuations. The only evidence that cavity pressure measurements are meaningful in this flow regime rests upon the agreement between measured forces and the theoretical calculations of $\mathrm{Wu}$ for two sharp-edged profiles (7). In these experiments, wherever it was found possible to measure the cavity pressure correctly, the experimental forces and the theoretical forces for this value $K_{k}$ were in good agreement.

\section{Cavitation Scaling}

For hydrofoil tests in partially cavitating or in fully cavitating flows, the cavitation number based on the cavity pressure is the primary scaling parameter. Therefore one could expect tunnel data taken in these flow regimes to be directly applicable to practical use for larger hydrofoils. However, for cavitation which starts on the hydrofoil the cavitation number for cavitation inception does show pronounced trends with free-stream velocity and perhaps with model size for flows without separation. For flows with separation the first signs of cavitation appear in the wake-vortex cores, and it would appear that this phenomenon is very sensitive to Reynolds number. If one is not concerned with noise generation, or with erosion of the hydrofoil in nonseparating flows, inception is of little importance because the forces on the profile are not greatly affected by the first signs of cavitation. For those cases in which the start of cavitation must be predicted accurately, it appears possible to determine inception points for prototype operations from water-tunnel tests if inception data are taken over a range of freestream velocities.

Recent tests of the NACA 4412 hydrofoil (5) showed the incipient cavitation number to increase continually with velocity over a range of speeds from 20 to $60 \mathrm{fps}$. The rate of increase of the incipient cavitation number with velocity became greater as the hydrofoil angle of attack was increased. Since the incipient cavitation number did not approach a constant value as the velocity was increased, it may be necessary to determine the incipient cavitation number over the range of test velocities available in the water tunnel and then extrapolate the results to the prototype speed. At no speed would one expect the incipient cavitation number to be greater than the magnitude of the minimum pressure coefficient on the profile as long as there is no flow separation. For flows with separation no such limit appears to exist.
There is an additional problem of the scaling of fullcavity flows which has not yet been touched upon specifically. If the designer wants to evaluate accurately the forces which act upon a profile at the higher cavitation numbers he must know the cavitation number $K_{k}$. Unfortunately, he does not know the actual cavity pressure in advance so that he has no way of calculating $K_{k}$. It may be that experimental results showing $K_{v}$ versus $K_{k}$, as in Fig. 4, are all he needs to complete the calculation. On the other hand there is no assurance that data of this sort, although valid for the model under one set of flow conditions, are also valid for the prototype under some other set of flow conditions. Certainly the dissolved air content of the water is one variable which could have an appreciable effect upon the results. In order to apply the results of water-tunnel tests to full-scale conditions with greater confidence, it would be of value to know more about the mechanics by which full-cavity flows are sustained, so that such factors as air diffusion and turbulent entrainment can be accounted for.

\section{Tunnel-Wall Effecis's}

The effects of tunnel walls on profile forces on noncavitating flows has been studied extensively by many investigators in aeronautics. On the other hand, the question of the effects of tunnel walls on full-cavity flows have not been explored so fully. The problem of wall effects for symmetrical two-dimensional cavity flows where the only force is a drag force, already has been investigated theoretically by Birkhoff, Plesset, and Simmons (10). They found that if the cavitation number is based on the cavity pressure, the drag coefficient for a given body is insensitive to the presence of the tunnel walls. Of course this result is not directly applicable to lifting hydrofoils in fully cavitating flows. For two sharp-edged hydrofoils it has been found experimentally (7) that the small discrepancies existing between theory and experiment were confined almost entirely to the lift force, and that the agreement for the drag force was excellent. These discrepancies in lift caused the lift-drag ratio for these profiles to have a trend with cavitation number opposite to that predicted by theory. Whether or not these small discrepancies can be ascribed definitely to the effects of tunnel walls must await the results of further investigation. ${ }^{4}$

\section{Hydroelastic Effects}

The introduction of the so-called supercavitating hydrofoil has brought with it some problems which result from the elasticity of the foil. These problems are not always important in hydrofoils designed for noncavitating flow because the fairly thick sections used for this purpose usually provide a sufficiently rigid structure. Super-

\footnotetext{
${ }^{4}$ After this paper had been submitted for publication, some new results on tunnel wall effects came to the authors' attention. They are contained in "Wall Effects in Supercavitating Flow Past Foils," by Hirsh Cohen and Yih-O Tu, Rensselaer Polytechnic Institute Math. Report No. 6, October, 1956. In this report, the effects of tunnel walls are determined by means of the linearized theory for $K=0$.
} 
cavitating hydrofoils, on the other hand, must be very thin in order not to interfere with the formation of the cavity at low attack angles. Therefore, they constitute comparatively flexible structures. From the point of view of two-dimensional tests in the High Speed Water Tunnel, the most important result of this increased hydrofoil flexibility is that appreciable spanwise twisting occurs. This twist gives balance indications which do not correspond to two-dimensional flow, and the data must be corrected for this effect. Fortunately, methods are at hand from the field of aeroelasticity (11) which can be applied to the present problem. In fact, the presence of the full cavity at the rear of the hydrofoil permits a greatly simplified treatment of the problem compared to conventional wing torsional-divergence calculations. For fully cavitating hydrofoils it seems reasonable to neglect all effects which might arise from a trailing vortex sheet in the calculations for spanwise twist.

For example, consider a supercavitating hydrofoil of span $l$ and chord $c$ mounted in the working section of the High Speed Water Tunnel, as shown in Fig. 1. From a structural point of view the model is a cantilever beam fixed at the end which attaches to the balance spindle. If this beam is regarded as being in free torsion, its deflection is given by

$$
\frac{d \alpha}{d x}=\frac{T(x)}{J G}
$$

where $\alpha$ is the local angle of twist, $x$ is the distance along the span measured from the built-in end of the beam. $T$ is the twisting moment about the center of twist of the profile, and JG is the torsional rigidity of the beam. The

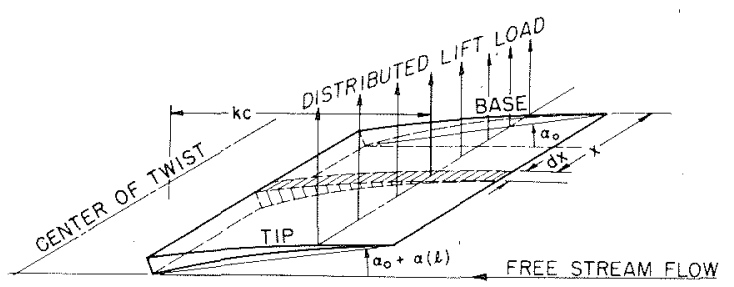

Fig. 6 Notations for hydrofoil twist corrections

beam and the forces acting on it are shown in Fig. 6 . The beam has a shape typical of a supercavitating hydrofoil with the center of twist a distance $k$ times the profile chord behind the center of pressure. The hydrofoil is now considered as being composed of a series of strips, as shown in Fig. 6. The section lift coefficient at the center of pressure of such a strip is given by

$$
C_{L}\left(\alpha_{0}+\alpha\right)=C_{L}\left(\alpha_{0}\right)+\left.\frac{\partial C_{L}}{\partial \alpha}\right|_{\alpha_{0}}+\ldots
$$

where $\alpha_{0}$ is the angle of attack at the built-in end of the foil and the local angle of attack is $\alpha_{0}+\alpha$. For most cases, the moment about the center of twist may be calculated with small error without considering the effect of the drag force. Accordingly, the element of moment per unit span is

$$
\frac{d T}{d x}=k c^{2} \frac{1}{2} \rho V^{2}\left[C_{L}\left(\alpha_{0}\right)+\left.\frac{\partial C_{L}}{\partial \alpha}\right|_{\alpha_{0}}\right]
$$

where $1 / 2\left(\rho V^{2}\right)$ is the dynamic pressure of the flow. From these results it follows that

$$
\frac{d^{2} \alpha}{d x^{2}}=-\frac{k c^{2}}{\mathrm{JG}} \frac{1}{2} \rho V_{0}^{2}\left[C_{L}\left(\alpha_{0}\right)+\left.\frac{\partial C_{L}}{\partial \alpha}\right|_{\alpha_{0}}\right]
$$

with the boundary conditions

$$
\alpha(0)=0, \alpha^{\prime}(l)=0 .
$$

The minus sign in the differential equation results from the fact that the rate of twist decreases as $x$ increases. The function.

$$
\alpha=\frac{q}{p^{2}}\left[\frac{\cos p(l-x)}{\cos p l}-1\right] \ldots \ldots \ldots
$$

where

$$
q=\frac{k c^{2}}{\mathrm{JG}} \frac{1}{2} \rho V^{2} C_{L}\left(\alpha_{0}\right), \text { and } p^{2}=\left.\frac{k c^{2}}{\mathrm{JG}} \frac{1}{2} \rho V^{2} \frac{\partial C_{L}}{\partial \alpha}\right|_{\alpha_{0}}
$$

satisfies the differential equation and the boundary conditions. The total lift on the hydrofoil may now be obtained in the form

$$
\frac{\mathrm{L}}{1 / 2 \rho V c l}=C_{L}\left(\alpha_{0}\right) \frac{\operatorname{Tan} p l}{p l}
$$

or in the alternate form

$$
\frac{\text { Measured lift coefficient }}{\text { Section lift coefficient }}=\frac{\operatorname{Tan} p l}{p l} \ldots . .
$$

where all coefficients are referred to the attack angle $\alpha_{0}$ set at the base. A similar result may be derived for the drag coefficient when it is represented by its Taylor expansion

$$
C_{D}=C_{C}\left(\alpha_{0}\right)+\left.\frac{\partial C_{D}}{\partial \alpha}\right|_{\alpha_{0}} \alpha+\ldots
$$

and in which the twist angle $\alpha$ is that due only to lift, as given in the foregoing.

In order to use Equation [2] for the correction of watertunnel data it is necessary to evaluate the quantity $p l$. The only quantity occurring in $p l$ which is difficult to determine theoretically with sufficient accuracy is the factor $\mathrm{k} / \mathrm{JG}$. Although this factor, in principle, can be determined with the help of the theory of elasticity, it is much simpler to evaluate it experimentally as follows: From Equation [1] it is easy to show that

$$
\cos p l=\frac{1}{1+\left(\frac{d C_{L}}{d \alpha} / C_{L}\right) \alpha(l)}
$$

where $\alpha(l)$ is the twist at the free end of the hydrofoil and both $C_{L}$ and $d C_{L} / d \alpha$ are evaluated at the angle $\alpha_{0}$. If, in addition to the force data, one also measures the twist $\alpha(l)$ for each data point, the quantity $p l$ may be found from the experimental data.

Associated with the static hydroelastic effects is the 


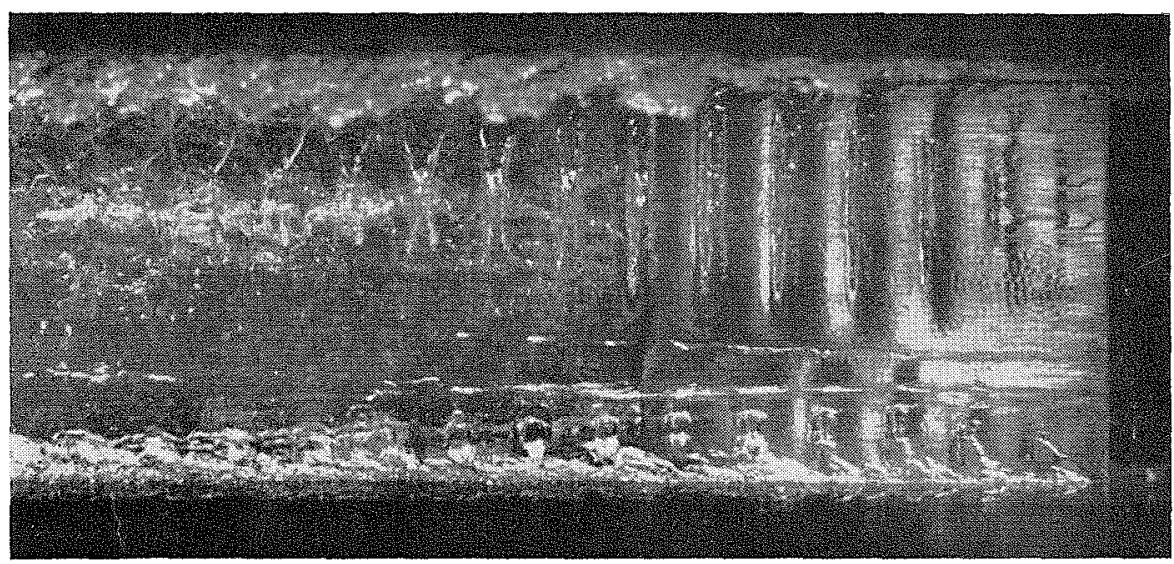

Fig. 7. Full cavity flow around a sharp-edged profile with vibrating leading edge. View looking down on plan of hydrofoil with flow from right to left

problem of hydrofoil vibration. For thin, flexible hydrofoils for which static twist corrections are necessary, bending and torsional vibrations may be expected. The characteristic frequencies of vibration are approximately the fundamental and harmonics of the bydrofoil. The excitation of the various modes depends on the amount of eavitation and end gap width. In the High Speed Water Tunnel the frequencies and modes of vibration appeared to be independent of angle of attack and free-stream velocity.

With leading-edge vibration in full cavity flow, waves are formed on the surface of the cavity. The amplitude of these waves may be such that the cavity is broken up into a train of short cavities separated by bands with no cavitation. Fig. 7 shows cavitation from a vibrating hydrofoil. Near the free end of the hydrofoil there is a region with a clear unbroken cavity which is a node point in the leading-edge vibration. Toward the free end from this point the wavelike cavities are shifted $180 \mathrm{deg}$ in phase relative to cavitation on the other side of the node. The frequency of vibration of the hydrofoil in Fig. 7 is approximately $800 \mathrm{cps}$.

The lift was not affected by hydrofoil vibration, but there was a small increase in drag, probably due to changes in the cavity configuration with vibration. With thin hydrofoils the amplitude of the leading edge vibration becomes quite large and may lead to fatigue failures of the foil. In addition, there is a considerable increase in noise level with foil vibration. Finally, it has been noted that cavitation from the end clearance gap is important in determining the mode of vibration of the hydrofoil model. For this reason, in studies of hydrofoil vibrations three-dimensional hydrofoil tests are needed to evaluate completely the effects of tip cavitation on hydrofoil vibration.

\section{Bibliography}

1 "Summary of Airfoil Data," by I. H. Abbott, A. E. von Doenhoff, and L. S. Stivers, Jr., NACA Report No. 824, 1945, appendix.
2 "The High Speed Water Tunnel Three Component Force Balance," by G. M. Hotz and J. T. McGraw, California Institute of Technology Hydrodynamics Laboratory Report No. 47-1, January, 1955.

3 "The Effect of Wall Interference Upon the Aerodynamic Characteristics of an Airfoil Spanning a Closed Throat Circular Wind Tunnel," by W. G. Vincenti and D. J. Grahm, NACA Technical Report 849, 1946.

4 "Tip Clearance Flows in Axial Flow Compressors and Pumps," by D. A. Rains, California Institute of Technology Hydrodynamics and Mechanical Engineering Laboratories Report No. 5, June, 1954.

5 "Water Tunnel Tests of NACA 4412 and Walchner Profile 7 Hydrofoils in Noncavitating and Cavitating Flows," by R. W. Kermeen, California Institute of Technology Hydrodynamics Laboratory Report No. 47-5, February, 1956.

6 "A Free Streamline Theory for Two-Dimensional Fully Cavitated Hydrofoils," by T. Yao-Tsu Wu, California Institute of Technology Hydrodynamics Laboratory Report No. 21-17, July, 1955.

7 "Experiments on Circular Arc and Flat Plate Hydrofoils in Noncavitating and Full Cavity Flows," by B. R. Parkin, California Institute of Technology Hydrodynamics Laboratory Report No. 47-6, February, 1956.

8 "Experimental and Theoretical Investigations of a Supercavitating Hydrofoil," by R. L. Waid and Z. M. Lindberg, California Institute of Technology Hydrodynamics Laboratory Report No. 47-8, in press.

9 "The Laws of Cavitation Bubbles at Axially Symmetric Bodies in a Flow," by H. Reichardt, Reports and Translations No. 776, Ministry of Aircraft Production, August, 1946, distributed in U. S. A. by Office of Naval Research, Navy Department, Washington, D. C.

10 "Wall Effects in Cavity Flow," by G. Birkhoff, M. S. Plesset, and N. Simmons, Part I, Quarterly of Applied Mathematics, vol. 8, no. 2, 1950, p. 151; Part II, vol. 9, no. 4,1952 , p. 413.

11 "Introduction to the Study of Aircraft Vibration and Flutter," by R. H. Scanlan and R. Rosenbaum, The Macmillan Company, New York, N. Y., 1951, Ch. 14. 